\section{P151 DEMYELINATION WITH AUTOIMMUNE FEATURES (DAF) - RESULTS FROM THE ATTIKON COHORT}

'Dionysis Nikolopoulos, ${ }^{1}$ Antonis Fanouriakis, ${ }^{2}$ Dimitris Kitsos, ${ }^{3}$ Matilda Papathanasiou, ${ }^{2}$ Maria Chondrogianni, ${ }^{1}$ Panagiotis Garatziotis, ${ }^{1}$ Antigone Pieta, ${ }^{4}$ Triantafyllos Doskas, ${ }^{2}$ Konstantinos Voumvourakis, ${ }^{1}$ Dimitrios Boumpas. ${ }^{1}$ Rheumatology and Clinical Immunology Unit, 'Attikon' University Hospital, Athens; ${ }^{2}$ Dept. of Neurology, 'Attikon' University Hospital, Athens; ${ }^{3}$ Dept. of Radiology, 'Attikon' University Hospital, Athens; ${ }^{4}$ Dept. of Neurology, Athens Naval Hospital, Athens, Greece

\subsection{6/lupus-2020-eurolupus. 194}

Background Patients with demyelination may manifest features of autoimmune rheumatic diseases. We evaluated patients presenting with a demyelinating syndrome and clinical or autoantibody evidence for an underlying connective tissue disease (CTD).

Methods Patients with clinical and/or imaging evidence of demyelination referred to a rheumatology unit. Diagnoses of multiple sclerosis (MS) and systemic lupus erythematosus (SLE) were made according to McDonald and SLICC classification criteria, respectively. Patients with features of CTD not fulfilling criteria for a specific disease were labelled as demyelination with autoimmune features (DAF). Demographics, clini$\mathrm{cal} /$ serological/imaging data and treatments were recorded at every visit, following multidisciplinary evaluation (neurology, rheumatology, neuroimaging).

Results Sixty-five patients $(n=65)$ were included in the study [93.8\% female, mean (SD) age at first demyelinating episode 37.3 (11.8) and median (IQR) duration to last follow-up 4 (7) years]. Rheumatologic clinical manifestations and

\begin{tabular}{|c|c|c|c|}
\hline Items & $\begin{array}{l}\text { All patients } \\
(\mathrm{N}=65)\end{array}$ & $\begin{array}{l}\text { MS } \\
(\mathrm{N}=32)\end{array}$ & $\begin{array}{l}\mathrm{DAF} \\
(\mathrm{N}=22)\end{array}$ \\
\hline Acute cutaneous lupus, n (\%) & $40(61.5)$ & $21(65.6)$ & $10(45.4)$ \\
\hline Chronic cutaneous lupus, $\mathrm{n}(\%)$ & $1(1.5)$ & $1(3.1)$ & $0(0)$ \\
\hline Malar erythema, n (\%) & $25(38.5)$ & $13(40.6)$ & $6(27.3)$ \\
\hline Photosensitivity, n (\%) & $35(53.8)$ & $17(53.1)$ & $9(40.9)$ \\
\hline Arthritis, n (\%) & $56(86.2)$ & $17(53.1)$ & $18(81.8)$ \\
\hline Non-scarring alopecia, n (\%) & $21(32.3)$ & $7(31.8)$ & $9(40.9)$ \\
\hline Oral/nasal ulcers, n (\%) & $14(21.5)$ & $8(25)$ & $5(22.7)$ \\
\hline Serositis, n (\%) & $2(3.1)$ & $0(0)$ & $0(0)$ \\
\hline Nephritis, n (\%) & $1(1.5)$ & $1(3.1)$ & $0(0)$ \\
\hline Leukopenia, n (\%) & $11(16.9)$ & $6(18.8)$ & $3(13.6)$ \\
\hline Thrombocytopenia, n (\%) & $2(3.1)$ & $1(3.1)$ & $0(0)$ \\
\hline $\begin{array}{l}\text { Cranial neuropathy (other than optic nerve), } \\
\text { n (\%) }\end{array}$ & $12(18.5)$ & $6(18.8)$ & $1(4.5)$ \\
\hline Seizures, $n(\%)$ & $2(3.1)$ & $1(3.1)$ & $4(18.2)$ \\
\hline Fever, $\mathrm{n}(\%)$ & $7(10.8)$ & $4(12.5)$ & $1(4.5)$ \\
\hline Sicca, n(\%) & $14(21.5)$ & $11(34.4)$ & $1(4.5)$ \\
\hline Raynaud's, n(\%) & $18(27.7)$ & $9(28.1)$ & $5(22.7)$ \\
\hline Livedo reticularis, $\mathrm{n}(\%)$ & $6(9.2)$ & $3(9.4)$ & $2(9.1)$ \\
\hline Antinuclear antibodies (ANA), n(\%) & $49(75.4)$ & $23(71.9)$ & $17(77.3)$ \\
\hline Anti-dsDNA, n(\%) & $5(7.7)$ & $1(3.1)$ & $1(4.5)$ \\
\hline Low complement, n(\%) (C3/C4) & $13(20)$ & $4(12.5)$ & $3(13.6)$ \\
\hline Anti-SSA, n(\%) & $10(15.4)$ & $5(15.6)$ & $4(18.2)$ \\
\hline Anti-SSB, n(\%) & $5(7.7)$ & $2(6.3)$ & $1(4.5)$ \\
\hline Anti-RNP, n(\%) & $3(4.6)$ & $2(6.3)$ & $1(4.5)$ \\
\hline Antiphospholipids antibodies (aPL), n(\%) & $11(16.9)$ & $6(18.8)$ & $4(18.2)$ \\
\hline CSF presence of oligoclonal bands, $n(\%)$ & $20(30.8)$ & $18(56.3)$ & $1(4.5)$ \\
\hline CSF positive $\lg \mathrm{G}$ index, $\mathrm{n}(\%)$ & $36(55.4)$ & $17(53.1)$ & $6(27.3)$ \\
\hline
\end{tabular}

autoantibodies of all patients are summarized in table 1. Fiftytwo patients had lesions in the brain $(80 \%), 32$ in the spinal cord $(49.2 \%)$ and 5 in the brainstem (7.7\%), while 17 developed optic neuritis (26.2\%). Among the 65 patients, at last follow-up, 32 patients (49.2\%) had fulfilled diagnostic criteria for MS with 11 patients (34.4)) diagnosed as overlap between SLE and MS and 16 patients (50\%) had CTD features not fulfilling criteria for any known CTD. Of patients with demyelinating syndrome not fulfilling criteria for MS $(n=33)$, 7 patients $(21.2 \%)$ had SLE while 22 patients $(66.7 \%)$ were classified as DAF. Most common features of DAF patients were ANA, arthritis and mucocutaneous features of SLE.

Conclusions Among patients with demyelination and features of CTD a significant number of patients do not fulfil criteria for either MS or SLE. These patients exhibit lupus-like autoimmune features and may represent a distinct group of patients.

\section{P152 EPIDEMIOLOGY AND PRACTICE PATTERNS IN THE MANAGEMENT OF LUPUS NEPHRITIS IN AUSTRALIA}

${ }^{1}$ Richard Phoon, ${ }^{2}$ Fiona Brown, ${ }^{3}$ Toby Coates, ${ }^{4}$ Sharon Ford, ${ }^{5}$ Ashley Irish, ${ }^{6}$ Nicole Isbel, ${ }^{7}$ Mark Thomas, ${ }^{8}$ Kate Wyburn. ${ }^{1}$ Westmead Hospital, Sydney; ${ }^{2}$ Monash Medical Centre, Melbourne; ${ }^{3}$ Royal Adelaide Hospital, Adelaide; ${ }^{4}$ St Vincent's Hospital, Melbourne; ${ }^{5}$ Fiona Stanley Hospital, Perth; ${ }^{6}$ Princess Alexandra Hospital, Brisbane; ${ }^{7}$ Royal Perth Hospital, Perth; ${ }^{8}$ Royal Prince Alfred Hospital, Sydney, Australia

\subsection{6/lupus-2020-eurolupus. 195}

Background In Australia, Lupus Nephritis (LN) affects a culturally diverse population of patients. We established the Lupus Nephritis Australian Registry (LUNAR) to better understand the population of patients receiving Myfortic and other immunosuppressants, and to analyse the safety and efficacy of the treatments they received.

Methods A non-interventional, multicentre, registry of patients with biopsy-proven ISN/RPS class III, IV or $\mathrm{V}$ LN, treated with Myfortic or other immunosuppressants, was established. We collected baseline demographic and 6-monthly follow-up data over a 5-year period (2013-2018), including clinical data, laboratory tests and safety outcomes.

Results 149 patients were enrolled in LUNAR across 8 sites, with $83.7 \%$ female and a mean age of 38.8 years. Most patients were Caucasian (45.2\%) - patients of Asian ethnicity (29.6\%) and Aboriginal/Torres Strait Islander or Maori/Pacific Islander descent $(10.4 \%)$ were significantly over-represented compared to the general population. The mean and median duration of SLE was 8.6 and 6 years, respectively (range 0-42 years), and of LN was 5.4 and 3.9 years, respectively (range 0-30 years). Most patients had class IV LN on their initial kidney biopsy (59.3\%), with $21.5 \%$ having class III and $11.1 \%$ having class V LN. Immunosuppressants used prior to screening included corticosteroids (68.9\%), mycophenolate mofetil ((MMF) 61.5\%), Myfortic (41.5\%) and cyclophosphamide (32.6\%). At enrolment or during the study, most patients were treated with Myfortic (76.3\%), MMF (65.2\%) and/or hydroxychloroquine (67.4\%). Rituximab and azathioprine were each started in $8.9 \%$ of patients during the study period. Kidney function was stable/improved for most patients over the study period and mycophenolate-based therapy was well-tolerated.

Conclusions LUNAR is the first study outlining the demographics, outcomes and practice patterns in the management of patients with $\mathrm{LN}$ in Australia. 